Itinéraires Itinéraires

Littérature, textes, cultures

2017-1 | 2018

Biographie et fiction

\title{
Tina Modotti d'Ángel De la Calle : un imaginaire (auto)(bio)graphique
}

(Auto)(bio)graphical Imagination in Ángel De la Calle's Tina Modotti

\section{Marie-Caroline Leroux}

\section{OpenEdition}

Journals

\section{Édition électronique}

URL : http://journals.openedition.org/itineraires/3739

DOI : $10.4000 /$ itineraires.3739

ISSN : 2427-920X

Éditeur

Pléiade

Référence électronique

Marie-Caroline Leroux, «Tina Modotti d'Ángel De la Calle : un imaginaire (auto)(bio)graphique », Itinéraires [En ligne], 2017-1 | 2018, mis en ligne le 15 février 2018, consulté le 02 mai 2019. URL http://journals.openedition.org/itineraires/3739; DOI : 10.4000/itineraires.3739

Ce document a été généré automatiquement le 2 mai 2019.

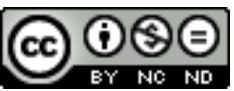

Itinéraires est mis à disposition selon les termes de la licence Creative Commons Attribution - Pas d'Utilisation Commerciale - Pas de Modification 4.0 International. 


\title{
Tina Modotti d'Ángel De la Calle : un imaginaire (auto)(bio)graphique
}

\author{
(Auto)(bio)graphical Imagination in Ángel De la Calle's Tina Modotti
}

\author{
Marie-Caroline Leroux
}

On examinera ici quelques aspects de la biographie dessinée Tina Modotti ${ }^{1}$, qui explore le parcours de la photographe italienne éponyme. Comme en atteste le titre original Modotti. Una mujer del siglo XX -, l'œuvre d'Ángel De la Calle ambitionne à la fois de dessiner les contours d'une vie et de la replacer dans son contexte historique : celui des grands combats idéologiques du siècle passé, dont Tina Modotti fut un témoin privilégié. La couverture de l'édition intégrale espagnole est rouge ; rouge aussi est l'engagement de la photographe, qui s'investit passionnément dans l'aventure communiste. Histoire d'un éveil au militantisme, ce récit lucide est aussi celui de la reprise en main du Komintern par Joseph Staline, des procès de Moscou et de la Grande Terreur. Borné par l'arrivée au Mexique de la jeune femme au bras de son mentor Edward Weston, en $1922^{2}$, et par sa mort mystérieuse à Mexico sur la banquette arrière d'un taxi, deux décennies plus tard, le parcours de Tina Modotti s'ouvre et se referme sur le même décor. Dans l'intervalle elle aura, au fil de son exil et de ses missions d'agent du Komintern, foulé le sol de l'Allemagne nazie, vécu à Moscou au plus fort des grandes purges et parcouru l'Espagne en guerre pour le compte du Secours rouge international.

Dans la mise en forme du biographique, le texte-image cultive la précision et la sobriété. Les récitatifs privilégient un cadrage factuel serré et la ligne graphique, assez austère, tend parfois à se faire oublier au fil de la lecture. Le primat de la dimension plastique est toutefois évident en un certain nombre d'occasions, comme peut l'être celui de la dimension littéraire - de la littérarité - dans la biographie textuelle. Cette alliance de la recherche esthétique et du souci de faire œuvre de connaissance ne fait guère débat dans le champ de la biographie. Plus généralement d'ailleurs, les orientations récentes de l'historiographie témoignent de l'intérêt pour l'articulation de ces deux visées. Ivan Jablonka fait ainsi de l'association des sciences sociales et de la création littéraire une impérieuse nécessité ${ }^{3}$. Ce postulat fort me semble un excellent point de départ pour 
l'étude d'une biographie qui ne veut renoncer ni à la rigueur, ni à la prise de position artistique.

3 La praxis biographique d'Ángel De la Calle rejoint au reste à plus d'un titre celle de Jablonka, qui place le "je" au cœur de la recherche historienne (2012 : 263-303). Dans cette œuvre consciente d'elle-même, l'auteur met en effet en scène sa recherche de la vérité de son personnage, sur un mode autobiographique, mais aussi - et c'est plus épineux autofictionnel ${ }^{4}$. Il faut ici rappeler que le recours à une double ligne narrative est fréquent dans la bande dessinée historique. Que le point de vue gémellé y passe par une figure d'auteur ou par un personnage de pure fiction, il traduit une proximité avec le sujet. La double trame est à ce titre particulièrement employée dans ces œuvres dont les auteurs s'attachent à sonder, au présent, des blessures historiques encore à vif, ou à explorer leur passé familial ${ }^{5}$. Le lien qui unit De la Calle à Modotti touche pour sa part à l'engagement politique, dans son association avec la pratique artistique. Revenant dans les séquences autobiographiques sur ses propres années de militantisme dans le sillage de l'écrivain Paco Ignacio Taibo II, il rappelle ainsi sa contribution comme dessinateur à une revue asturienne anarchiste. Cet engagement s'inscrit dans le cadre de la lutte de la gauche espagnole des années 1970 pour la restauration de la démocratie. L'auteur évoque abondamment son compagnonnage avec Taibo, notamment pour souligner leur acharnement à défendre le festival de littérature policière créé par le même Taibo en 1988 - la Semana Negra de Gijón - contre les assauts de la droite conservatrice, qui lors de la campagne pour les élections municipales de 1995 avait fait de la suppression de la Semana Negra l'un des points forts de son programme. Mais, et c'est là que les chemins du biographe et de la biographée se séparent, De la Calle n'occulte rien non plus du désenchantement qui s'empara de sa génération dans les années de l'après-Transition ${ }^{6}$ et de sa progressive prise de distance d'avec l'action politique. Modotti, pour sa part, reste fidèle au Parti communiste, au plus fort de la répression stalinienne. Elle ne renoncera à sa carte du Parti que quelques mois avant sa mort, du fait de son désaccord avec la signature du pacte germano-soviétique.

L'œuvre ne ressort pas, on l'aura déjà compris, à une catégorie discursive claire. Et cette hybridité constitutive invalide a priori toute tentative d'inscrire Tina Modotti dans le champ de la biographie ${ }^{7}$. C'est pourtant ainsi que nous prenons le parti de l'envisager ici, attendu que la ligne métabiographique est clairement circonscrite au plan scénaristique et visuel et nettement seconde dans l'économie narrative.

On s'attachera ici à déterminer dans quelle mesure et par quel biais cette biographie singulière devient scène du biographe, en examinant la manière dont l'imaginaire et la personne du biographe travaillent et trouent la matière biographique. On commencera par revenir sur le fonctionnement du niveau biographique, où malgré le recours à la première personne le récit s'efforce à l'objectivité ; et l'on s'intéressera également au rôle de l'imaginaire graphique dans le développement d'une forme de médiation documentaire. Le niveau métabiographique sera au cœur de la seconde partie de l'analyse. Parce que ce niveau fait l'objet de dérapages autofictionnels, il signe par instants une véritable entrée en régime de fiction. L'imaginaire trouve à s'y épanouir à travers le personnage d'Ángel, alter ego de l'auteur. Ce n'est qu'après avoir embrassé ces deux aspects de la bande dessinée - le biographique puis le métabiographique, dans ses deux états : autobiographique et autofictionnel - et à la lumière, cette fois, de la relation biographique, qu'on pourra percevoir la véritable portée des choix esthétiques d'Ángel De la Calle, qui s'expriment autant dans les hommages appuyés à la peinture engagée que 
dans l'alliance de la photographie et de la case. Dans cette biographie peu orthodoxe, en effet, seul le raccord identitaire biographe-biographée permet une véritable compréhension du rôle de l'imaginaire plastique.

\section{Médiation documentaire et façonnage de la matière biographique : les formes du biographique dans Tina Modotti}

6 La légitimation publique et éditoriale de la biographie dessinée s'assortit progressivement d'une légitimation d'ordre scientifique et l'intérêt des historiens pour la bande dessinée va grandissant ${ }^{8}$, la plupart s'accordant à considérer qu'elle peut participer d'une reconstitution aussi synthétique qu'efficace du passé, en autorisant le regard et la pensée à embrasser d'un seul mouvement le moment historique. Il s'en trouve même qui, sans renoncer à l'affirmation de la spécificité de leur discipline, constatent qu'elle peut contribuer à la production de connaissances historiographiques nouvelles (Jablonka 2014). L'ambition de l'auteur de Tina Modotti est plus modeste. Il procède par collecte et transmutation de données déjà exploitées et assume pleinement la nature seconde de son œuvre. Il affiche ainsi ses sources en de nombreuses occasions, usant pour ce faire du canal iconique autant que du canal textuel ${ }^{9}$. Ces passages font bien souvent office d'apparat critique pour le personnage d'auteur, qui commente volontiers le positionnement de tel ou tel biographe. Leur fonction essentielle consiste cependant à interroger le processus d'élaboration de l'œuvre. En ce sens la forme que prend cette biographie dessinée rappelle, en dépit de la légèreté affichée du ton, le «texte-enquête " que Jablonka appelle de ses vœux et l'intérêt que peut revêtir l'usage du "je" dans le raisonnement historien :

L'usage du « je » est une liberté épistémologique avant d'être un choix d'écriture. À l'histoire-résultat, on préférera - pour des raisons scientifiques - la véritéprocessus, c'est-à-dire la manière rationnelle, explicable, amendable, dont on a tendu vers un but. La question, l'enquête, la recherche, la démonstration sont les jalons du chemin cognitif. [...] Faute de s'intéresser aux secrets d'alcôve, aux portes dérobées et autres coulisses de l'Histoire, on peut faire visiter celles du livre luimême. (Jablonka 2014b : 295)

7 Le souci d'éclairer la fabrique de la biographie débouche sur un parti pris énonciatif du même ordre chez De la Calle. Dans nombre de biographies dessinées, la narration est résolument non interventionniste; le foyer d'énonciation reste flou et le récit déploie sa magie, interdisant au lecteur tout effet de distanciation. Rien de tel ici, puisque l'auteur s'exhibe de bout en bout comme récitant et devient même "narrateur actorialisé " (Groesteen 2011 : 106) dans le niveau iconique de la ligne métabiographique. L'approche par le «je» force le lecteur à s'arracher à la fascination du récit - à l'illusion biographique - et sa subjectivité assumée vaut pour invitation à la réflexion. C'est spécialement patent dans les épisodes de la ligne seconde où le personnage d'auteur croise le fer sur des questions politiques avec Taibo. Dans cet extrait, le dialogue est ainsi rendu à son vieil usage polémique : l'auteur en use pour contextualiser la controverse sur les circonstances de la mort de $\mathrm{T}$. Modotti. Au lecteur, en dernière instance, de se forger une opinion :

(Paco) Dire que Tina est morte assassinée est une interprétation tendancieuse de plus et une calomnie!

(Ángel) Paco! Une femme de seulement 46 ans, sans antécédents cardiaques... Elle 
serait morte d'une attaque?

(Paco) Donc, selon toi, c'est encore une conspiration stalinienne?

(Ángel) Non Paco, je veux savoir ce qui s'est passé, il y a tant de zones d'ombre dans la vie de Tina... Beaucoup trop!

(Paco) Mais tu vois des machinations et des complots de ses camarades et amis partout...

(Ángel) C'est ça... Et dans l'affaire Andreu Nin ou Trostky, on disait aussi que le KGB

n'avait rien à voir non plus... Ne sois pas naïf, Paco ! (129-9 et 130-1 à 6)

Leurs échanges nourris mettent par ailleurs à nu les ressorts du travail d'investigation. Ils éclairent en particulier les difficultés liées aux lacunes de la documentation. La négociation avec la lacune participe pleinement de l'opération biographique ${ }^{10}$, aussi le récitant multiplie-t-il les questions dans le corps de la biographie, sans s'essayer à y répondre. Et c'est aux modalisateurs qu'il recourt à l'heure d'évoquer le séjour clandestin de T. Modotti à New York, en 1939, alors qu'elle travaillait déjà pour le Komintern: «Durant un mois, Tina corrigea, semble-t-il, la traduction anglaise du livre autobiographique de son amie Constancia de la Mora ; «La mission de Tina dut être différente »; « On dit qu'à New York, elle vit son frère et sa sœur [...]. Ou peut-être pas » (230-7, 230-9 et 231-1).

9 Ces précautions prises, le bédéiste peut user des qualités propres au média pour s'essayer à une mise en perspective historique susceptible d'éclairer la trajectoire de son personnage. Au plan iconique, figures et décors relèvent le plus souvent de l'épure : ce n'est donc pas dans les détails du dessin qu'il faut chercher la restitution du moment historique, mais plutôt dans les récitatifs, qui peuvent prendre un tour relativement didactique. Le dessinateur réserve ses audaces graphiques à un nombre restreint de hauts-lieux de l'histoire de la première moitié du siècle. Ces vignettes spectaculaires imposent une scansion, structurent et balisent le parcours erratique de T. Modotti en des temps troublés. De loin en loin, elles octroient une respiration au lecteur engagé dans le récit factuel, en interrompant brièvement la continuité séquentielle. Observons l'édifice de la Loubianka, sinistre siège du KGB, à l'époque où la jeune femme réside à Moscou en tant qu'agent du Parti (fig. 1). 
Fig. 1. Planche $183^{11}$.

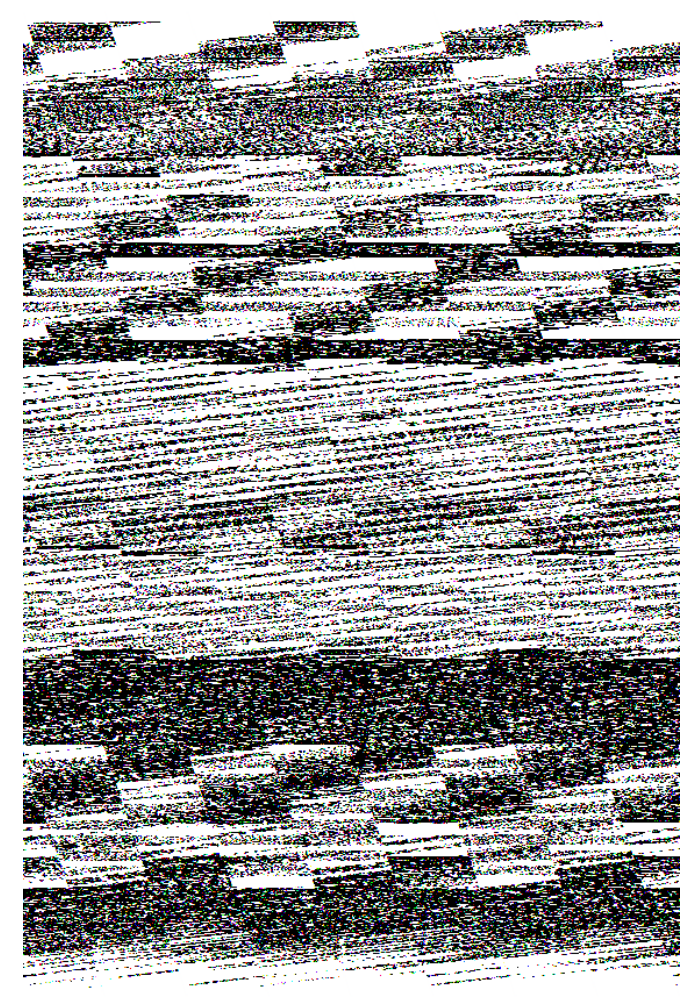

Dans cette case-planche, le lieu historique joue le rôle d'embrayeur du cadrage historien, qui catalyse à son tour le discours biographique, la translation s'effectuant dans le mouvement descendant de la lecture. La hauteur de la ligne d'horizon et l'effacement du ciel dans l'espace hypercadre ${ }^{12}$ mettent en relief la Loubianka, dont les contours dessinent la bordure supérieure de la vignette. La grisaille de la bâtisse s'étend à la place qui porte son nom, saturant complètement l'espace iconique avant de s'épancher dans une flaque sanglante, qui ne laisse aucun doute quant à l'intention critique du dessinateur. Dans la partie médiane, les cartouches, les portraits incrustés et leurs notices répondent à la nécessité d'une contextualisation précise. Teintés de subjectivité, ils reflètent également le désenchantement personnel de l'auteur face au dérapage de l'idéal communiste et enclenchent les interrogations relatives à la personne de Modotti : « Tina vécut à Moscou en ces temps amers et malsains. Fut-elle capable de voir ce que cachait la propagande officielle? / Les véritables croyants se doivent-ils d'être aveugles et sourds?» La silhouette de la biographée est déposée sur cet oppressant arrière-plan, vide des réponses à ces questions. Si la solidité du cadrage historique est indéniable, ce type de vignettes est une première occasion d'observer les tiraillements internes du texte-image, partagé entre le souci d'objectivation et l'intention critique.

11 Dans le cas du séjour de Modotti dans le Berlin des années 1930, la prise de position esthétique est plus notable. À l'évidence, le choix d'une mise en image forte vient ici compenser la timidité des récitatifs pour ce qui touche au rappel des circonstances historiques : le texte l'éludant à dessein pour continuer de dérouler le fil de la biographie, c'est à l'image qu'est dévolue l'évocation de l'atmosphère délétère de l'Allemagne de l'entre-deux-guerres. Le tableau d'époque donne lieu à des entorses caractérisées à la ligne graphique dominante. L'auteur, prenant le parti de l'artificialisation, introduit dans un certain nombre de vignettes des éléments empruntés à l'esthétique expressionniste. 
Ils prennent une forme furtive: des visages clownesques ou difformes jouent les admoniteurs au sein de cases d'apparence anodine. "Regardez mieux la case!", semblent-ils dire au lecteur embarqué dans le récit biographique, happé par les données visuelles et textuelles qui l'invitent à suivre la biographée dans les rues de Berlin, et à ne suivre qu'elle. Car le face-à-face qu'imposent au lecteur les figures de contact expressionnistes déposées au premier plan revêt bel et bien une fonction de mise en garde. Derrière la silhouette d'un estropié de guerre ou celle d'un homme jeté dehors à coups de pied, ces vignettes révèlent, dans les détails de l'arrière-plan, les séquelles du premier conflit mondial et la violence des répercussions de la crise économique (fig. 2).

Fig. 2. Planche 135, vignette 3.

\section{TAMBIEN ENCONTRÒ QUE HABIA MASS DE QUINIENTOS \\ ESTUDIOS FOTOGRAFICOS EN BERLIN. COMPETIR CON LOS CAPACES PROFESIONALES GERMANOS NO SERIA FACIL.}

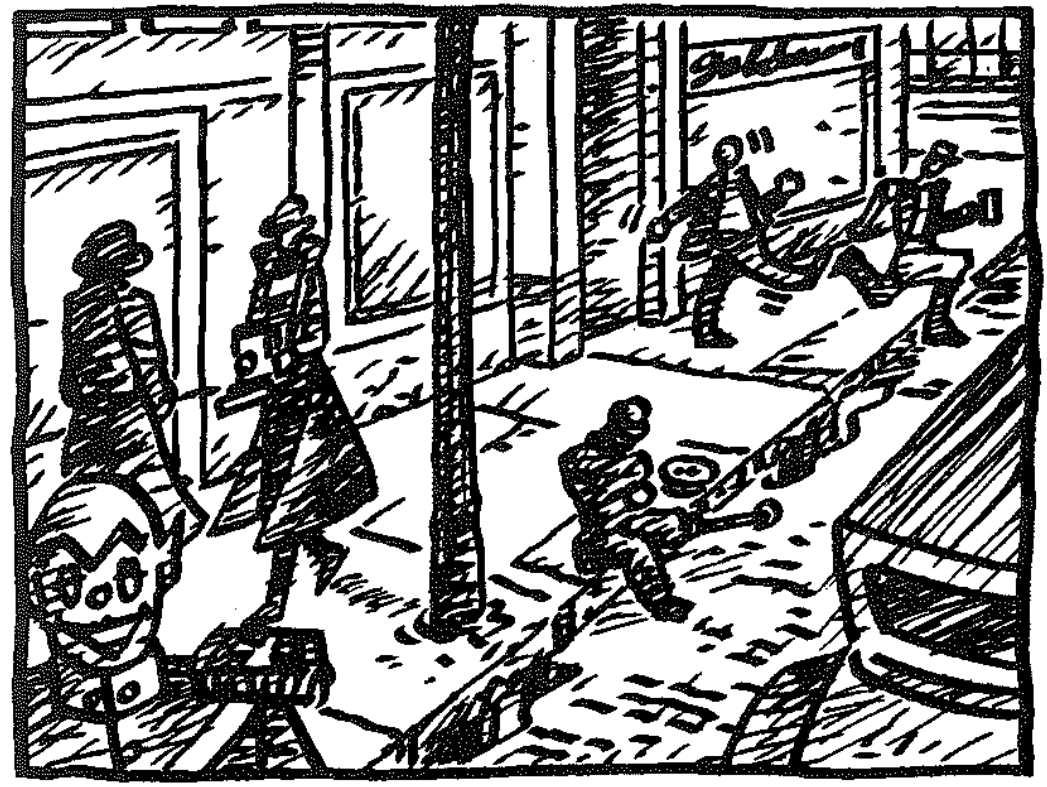

L'impact de certaines cases berlinoises, et celle-ci en est un bon exemple, est décuplé par la parfaite autonomisation de ces premiers ou de ces arrière-plans menaçants par rapport à l'action narrative. Le personnage principal vaque à ses occupations, ignorant leur présence. Négligés et par le narrateur et par les personnages, ils n'en attirent que davantage l'attention. Figures mais aussi décors contribuent donc à traduire l'époque par la monstration d'une humanité grotesque et macabre ou par la déstructuration d'un espace urbain réduit à des lignes brisées ${ }^{13}$, dans une veine qui rappelle l'œuvre peinte d'Otto Dix et les gravures de Lyonel Feininger.

13 Ces références à l'expressionnisme continuent, en apparence du moins, de relever d'une forme de médiation documentaire: l'intericonicité nourrit le tableau d'époque, et l'auteur atteint par ce biais à une synthèse de la noirceur du temps. Il nous faudra pourtant y revenir dans la suite de l'analyse, car elles s'ajoutent à d'autres jeux de citations iconiques qui pour leur part engagent fortement la subjectivité du bédéiste - comme Espagnol, comme militant de gauche, comme artiste -, comme ce dialogue avec le Guernica de Pablo Picasso, qui sert d'appui à la séquence consacrée à la guerre 
d'Espagne (217 à 224). Le dialogue artistique interroge en réalité, nous le verrons, les limites de l'exercice de funambulisme auquel De la Calle se livre dans cette biographie.

Considérons enfin le modelage des données biographiques proprement dites. Malgré la revendication de non-fictionnalité qui préside à son entreprise, tout biographe se soumet, au même titre que l'historien, aux lois de la poétique. Il lui faut produire un « effet de vécu » (Dosse 2010 : xvI) susceptible de doter l'œuvre d'une certaine intensité narrative. La multimodalité propre au neuvième art autorise ici un traitement différencié du vrai et du vraisemblable. On observe en effet que la mise en intrigue de la matière biographique s'opère essentiellement dans le niveau iconique : au dessin et au dialogue, l'anecdote et l'effet de vécu ; les récitatifs supportant l'essentiel des données factuelles.

Dans l'ensemble, le façonnage du fait biographique demeure minime. L'opération de fabulation se réalise dans le respect de l'«éthique biographique ${ }^{14}$ ». On en trouve une excellente illustration dans les pages qui relatent la mort du compagnon de Modotti, Julio Antonio Mella (107 à 110), dans lesquelles le personnage d'auteur expose deux théories distinctes sur son exécution, renonçant ainsi à négocier avec l'absence de données factuelles pour exhiber les brèches du savoir biographique. Conscient de ces lacunes, l'auteur n'aspire nullement à une restitution de type totalisante.

Quant au portrait de Modotti, il est tracé en creux. Dans la mesure où l'auteur renonce à évoquer la vie intérieure de son personnage, il échappe de fait et à la tentation téléologique et à celle d'assigner à son sujet une identité monolithique, parfaitement stable (Bourdieu 1986: 70). Le récitant, véritable surmoi biographique, exerce une fonction de contrôle permanente sur le récit. Les cartouches renvoient ainsi systématiquement la biographée à son énigmatique altérité. Le narrateur y procède par questions: "À quel moment perdit-elle la foi en l'art comme arme pour changer le monde? » (154). Pendant ce temps, le récit iconique assure le maintien du continuum narratif. L'hybridité du média, pourtant, aide à redonner forme au sujet biographique, car le bédéiste peut se soustraire au poids des mots et à leur impudeur en recourant au visuel pour suggérer l'épaisseur du personnage. L'inscription de Modotti dans de larges paysages ou des horizons urbains évasifs est récurrente, et la gestion de l'espace interne de ces vignettes, majoritairement silencieuses, dévoile une nette intentionnalité. Disproportionnés au regard de la silhouette gracile qui les hante, ces arrière-plans dégagent souvent une forte impression d'artificialité et semblent impuissants à fusionner avec elle dans un même pan de réalité iconique. La distance méditative qui se creuse entre le personnage et le paysage suggère le déploiement de cette intériorité que le biographe s'interdit de sonder (fig. 3). 
Fig. 3. Planche 200, vignette 1.

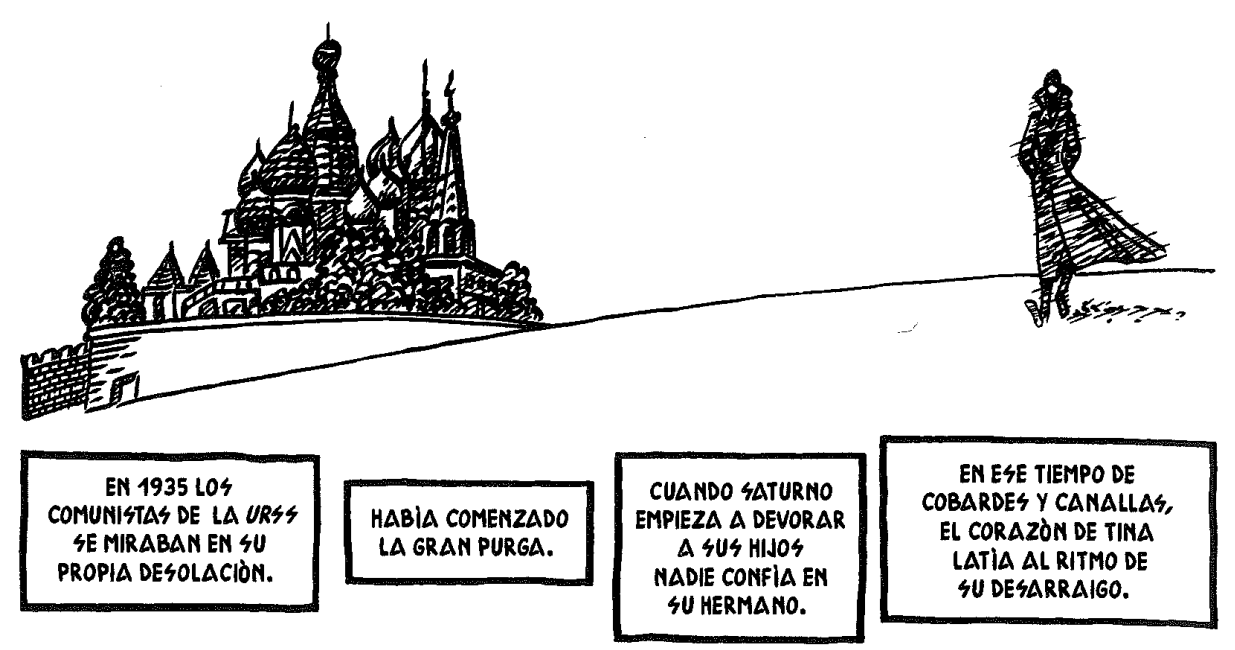

De loin en loin, la charge connotative du texte et, plus encore, de l'image, occasionne donc une brève suspension de la valeur de vérité de l'énoncé biographique. Cela ne fait que nous ramener à l'ambivalence constitutive du mode biographique, toujours écartelé entre visée scientifique et visée esthétique, mise à distance et partialité assumée. L'ambiguïté que cultivent les épisodes autobiographiques et leurs excroissances autofictionnelles est d'une autre nature : elle touche à la relation biographique.

\section{Le métabiographique : de l'autobiographie à l'autofiction}

Entre adhésion affective et distance critique, la relation du biographe à son sujet se traduit souvent en termes de possession ${ }^{15}$. Dans Tina Modotti, les interventions de l'instance narrative n'occultent rien de ce rapprochement fusionnel, de cette intersubjectivité qui s'exprime dans un riche éventail de nuances, depuis les récitatifs discrètement colorés d'affectivité jusqu'à l'épilogue de l'édition espagnole élargie, où le personnage d'Ángel vient déposer un exemplaire de son œuvre sur la tombe de Modotti : "Ce livre, Tina, conclut-il, c'est ton histoire et c'est aussi, un peu, ma vie » (De la Calle 2011a: 285) ${ }^{16}$. Le «je " n'a donc pas simplement vocation à localiser la parole du biographe, à retracer les étapes de son investigation et à rassembler, sinon à produire, des connaissances sur son sujet. Il est aussi profondément introspectif. La ligne seconde, qui s'affiche comme autobiographique, supporte l'essentiel de cette charge réflexive.

La co-présence de la biographée et de son biographe éclaire les ressorts de la relation qui les unit, et en particulier la question de l'engagement politique. Interrogé par une journaliste sur le pourquoi du choix de son sujet, De la Calle a cette réponse: «Parce qu'elle a combattu pour des causes justes, sous de mauvais drapeaux » (De la Calle 2011a : $280)^{17}$. Commun dénominateur du couple biographique, le militantisme adopte chez l'un et l'autre des formes très distinctes. En 1930, Modotti est expulsée du Mexique; elle se consacre dès lors exclusivement à sa mission au sein du Secours rouge international, un 
organisme humanitaire de soutien aux ouvriers, aux prisonniers et aux exilés politiques. Voilà pour la cause. Quant au drapeau, c'est bien entendu celui du Parti communiste. De la Calle, on l'a vu, interroge l'aveuglement dogmatique de son personnage, à qui il reproche de n'avoir pas su s'éloigner de la ligne officielle du Parti communiste. Les leçons du passé et l'érosion des idéologies limitent clairement l'identification du biographe avec son sujet. Et l'on mesure la distance qui les sépare au déplacement de la lutte politique, progressivement portée sur un terrain exclusivement culturel par l'auteur et son ami Taibo, avec la création de la Semana Negra.

20 C'est au fil de leurs dialogues que se fait le plus manifestement jour le désenchantement d'une modernité en crise. Mais puisque c'est ici la fictionnalisation qui nous occupe, laissons de côté les échanges ordinaires des deux comparses et tournons-nous vers ces épisodes autofictionnels qui suspendent brutalement l'illusion référentielle et ouvrent une nouvelle ligne de fracture dans le récit, dans une strate que le lecteur identifiait jusqu'alors comme autobiographique. Ángel et Paco sont en déplacement professionnel et le récit suit paisiblement son cours lorsque tout dérape, à deux reprises. Dans ces pages hallucinées, en effet, Ángel assiste à l'affrontement de deux super-héros décrépits - un Batman trostkyste et un Superman staliniste -, engagés dans une controverse doctrinale dans une chambre d'hôtel contigüe à celle qu'il partage avec Paco.

21 La proposition autofictionnelle n'a rien de frivole, car le bédéiste ne se contente pas de clins d'œil aux codes et aux personnages des comicbooks de super-héros. L'altération de l'archétype s'accompagne d'une subversion de leur vocation idéologique. Captain America, Superman et Batman luttaient contre les forces du mal, et celles-ci, après avoir été incarnées par les nazis, le furent par le monstre soviétique dans les années de la guerre froide. La sarcastique inversion de ce schéma - la transformation de Batman et Superman en hérauts du communisme - illustre la prise de distance de l'auteur d'avec tous les dogmatismes et, en particulier, d'avec le radicalisme de T. Modotti. Sommé par les super-héros de procéder à son propre examen de conscience politique, Ángel plaide pour la circonspection :

(Batman) Alors comme ça, vous ne pensez pas que le mal doit être extirpé à la racine et partout en même temps ? Vous croyez à la compromission?

(Ángel) Croire... ? Je crois aux femmes mortes vingt ans avant ma naissance... Je crois en la confusion... (207-8 et 9)

Relation infiniment paradoxale que celle-là: par-delà le désaccord idéologique, le personnage d'auteur réaffirme ses affinités avec la photographe.

Car la chimère qu'il poursuit est avant tout celle de la fusion avec son sujet, et l'imaginaire biographique est ouvertement au service de cette aspiration. Au cours de sa recherche, l'avatar de l'auteur place ses pas dans ceux du sujet biographique. Les bégaiements iconiques qui en résultent ${ }^{18}$ matérialisent la force du lien qui les unit. Les lieux arpentés par Modotti sont ce à quoi il s'arrime dans sa quête de la vérité intime de son personnage ${ }^{19}$, mais il est naturellement confronté aux limites de leur pouvoir d'évocation: les mutations de l'espace urbain lui signifient l'impossibilité à réancrer spatialement l'assassinat du révolutionnaire J. A. Mella (106), tandis que, dans les rues de Gijón, la réticence des lieux historiques s'exprime dans la mention de «l'insaisissable fantôme " de Tina (212-1). La tombe de la photographe fait exception à ce verrouillage. Rien là de surprenant, dans la mesure où la scène du cimetière (127 à 129) signe probablement la rencontre inaugurale entre le biographe et son sujet, par un après-midi des années 1990. Dans sa matérialité froide et crue, la tombe apparaît en tout cas à Ángel comme une forme de Tina. Et l'imaginaire se met en branle : 
[C]e minuscule tombeau me parla.../ Il me parla de ma génération amnésique, des défaites mal assumées et de la nécessité de se souvenir. / D'un lieu quelconque, séminal ou océanique, je sentis que Tina me demandait: "Qui raconte mon histoire ? ».../... « Qui la racontera »? (129-6,7,8).

$\mathrm{Au}$ point que lorsque le personnage caresse le profil de pierre gravé sur la dalle, la photographe semble un instant se matérialiser (fig. 4).

Fig. 4. Planche 126, strip 2

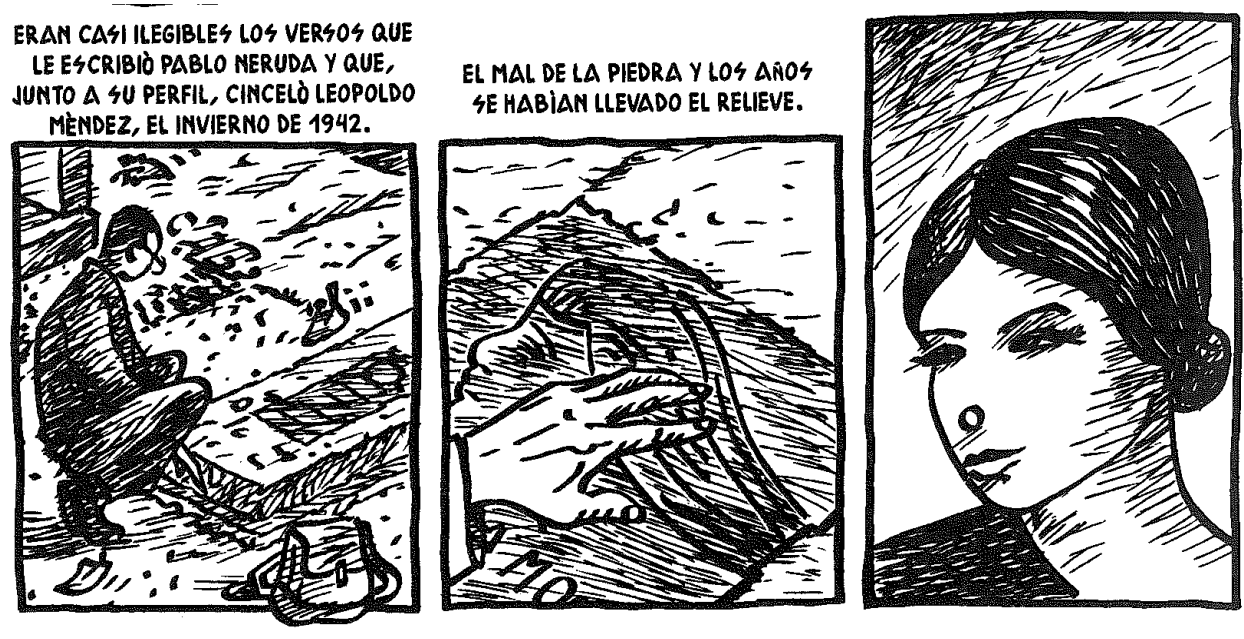

La vision, fugace, quasi subliminale, tient dans une case. La survenue de la figure de la biographée, en pleine ligne seconde, signe, en apparence du moins, une violation des frontières narratives. L'attitude de Modotti, pourtant, a tout de la pose. Et le lecteur ne tarde pas à y reconnaître l'un de ces innombrables clichés redessinés disséminés dans l'œuvre : il s'agit en fait là d'un des portraits de Modotti réalisés par Edward Weston dans les années 1920. À ce stade, on en reste donc au seuil de la métalepse.

Ce seuil est franchi au cours de la séquence finale, qui laisse s'épanouir l'imaginaire (auto) (bio)graphique. Car dans cette authentique zone de $\mathrm{f}(\mathrm{r})$ iction convergent tout à la fois le temps de Tina et le temps d'Ángel, la réalité et le fantasme. Le face-à-face du couple biographique se concrétise à Gijón, dans la rue Tina Modotti. Mais le lieu de la rencontre, plus que cette rue, c'est la case : avec les moyens propres à son art, De la Calle fait de l'espace dessiné l'interface où s'apparient les deux plans de la réalité bédéistique, dans une métalepse qui n'a plus rien de disruptif : Modotti lui apparait, au présent, dans une muette épiphanie (fig. 5). 


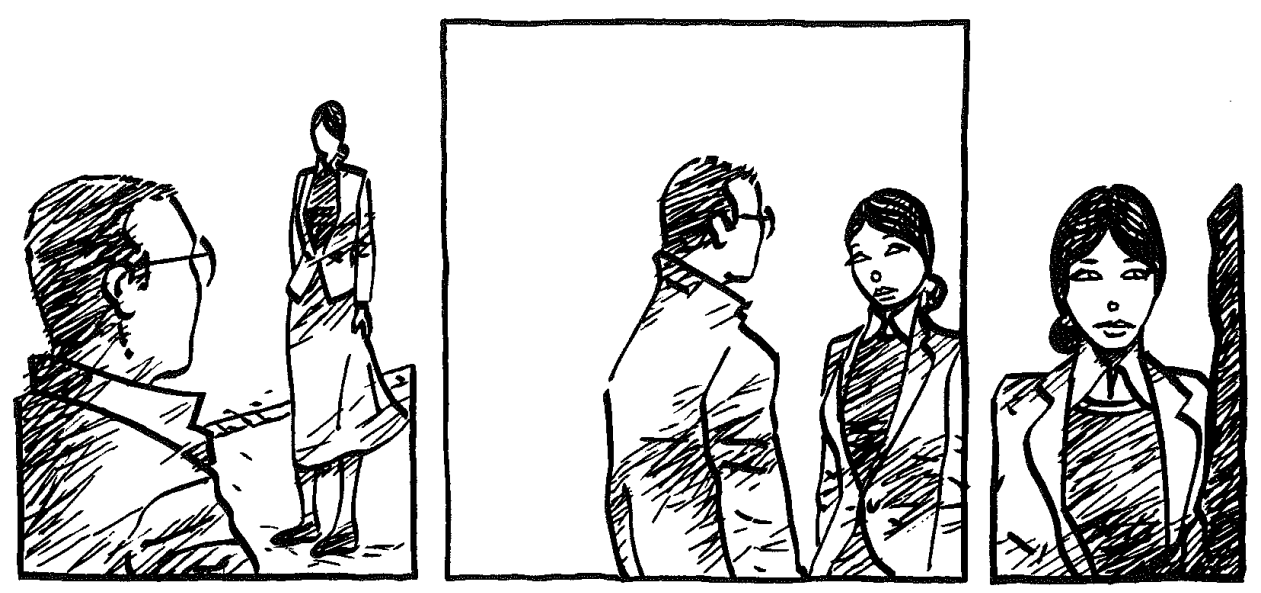
photographie par Modotti (fig. 6) et qui offre un bel exemple de l'importance que revêt pour l'auteur la question de la pratique artistique. La subjectivité du biographe s'y exprime tout entière dans le niveau iconique. Le visuel, en effet, désavoue ici le textuel pour offrir une vision ouvertement contre-factuelle de ce renoncement. Une vision davantage en accord avec la sensibilité de l'auteur, qui opte pour une version teintée de mélancolie et de solennité, à même de compenser, peut-être, la raideur militante de la nouvelle Tina, déterminée à se consacrer exclusivement à sa mission politique. 
Fig. 6. Planche 166, strip 2.
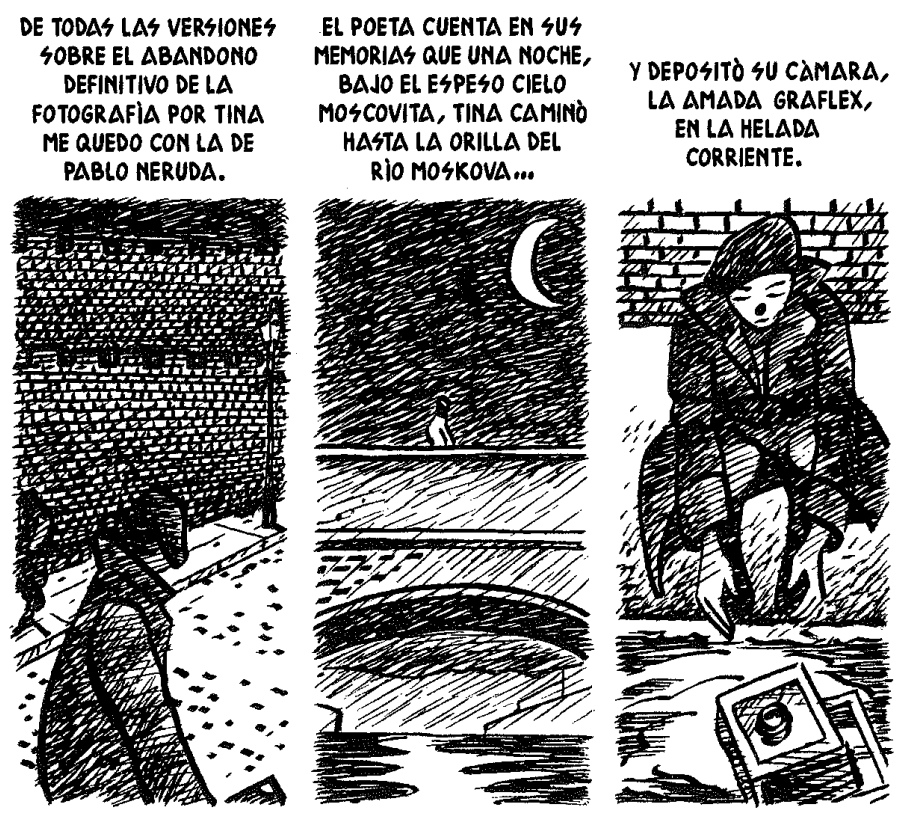

NO PARECE REALISTA

QUE OCURRIESE ASI...

IPERO QUE HERMOSO HUBIESE SIDO!

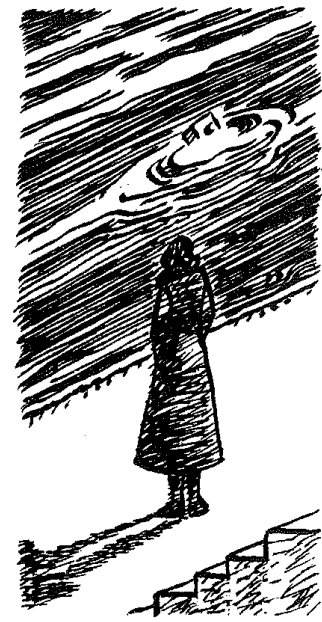
visuelle " (Groesteen 1999: 8) de la bande dessinée : il y a toutes les chances pour que le halo de romantisme dont est baignée la version dessinée influe davantage sur le lecteur que le dernier récitatif, tout soucieux que soit ce dernier de dénoncer la feintise.

30 Si le renoncement à la photographie de Modotti a pour De la Calle des airs de désertion, s'il éprouve la nécessité de le poétiser pour lui donner un sens, c'est que l'art à ses yeux est geste politique. Il y a là, sans doute, une vraie pierre d'achoppement pour ce qui touche aux limites de l'identification du biographe à sa biographée, car le bédéiste ne cède rien sur ce terrain, malgré le désenchantement : l'art est une arme, et peut-être la seule qui vaille encore.

31 Si donc De la Calle s'interroge sur la perte de la « foi en l'art comme arme pour changer le monde " de la photographe italienne (154-7), il proclame vigoureusement la sienne lorsqu'il réinvestit Guernica et un certain nombre d'œuvres d'art majeures du début du Xx e siècle. C'est en ce sens que les entorses à l'effet de réel bédéistique évoquées dans la première partie constituent une modalité essentielle de l'imaginaire graphique à l'œuvre dans Tina Modotti. Recourir à un type de figuration expressionniste pour décrire l'atmosphère de l'Allemagne des années 1930 et la montée du nazisme, c'est se souvenir que ce courant est né dans un temps de crise et d'angoisse, à l'aube de la Première Guerre mondiale, et se souvenir aussi qu'il a par la suite incarné, pour le III Reich, ce que l'« art dégénéré » avait produit de pire. Ce en quoi les emprunts du dessinateur à l'esthétique expressionniste sont assez ironiques. L'opération à laquelle se livre l'auteur ne consiste donc pas seulement à réinsérer les œuvres dans leur contexte de production, mais à les rendre à leur combativité et à leur potentiel subversif.

Le statut du bloc consacré à la guerre d'Espagne et à Guernica - tableau réalisé pour l'Exposition universelle de 1937 et dont le principal objectif était d'attirer l'attention du monde sur ce conflit et ses enjeux - est à ce titre tout à fait singulier. 

l'espace tabulaire en deux strips autonomes, assied, au long de ces huit pages, un code de lecture qui rompt avec le gaufrier. Au sinueux itinéraire de lecture conventionnel (de gauche à droite et de haut en bas), se substitue une lecture étagée au long cours : dans le bandeau inférieur se trouvent résumées trois années de guerre tandis que le bandeau supérieur rend hommage aux artistes qui s'engagèrent aux côtés de la République, en la personne d'un peintre, Pablo Picasso, et d'un poète, César Vallejo. d'histoire et condensé des péripéties de Modotti dans la guerre civile. Le propos s'y fait dense, nerveux, pour embrasser le conflit dans sa globalité : le découpage en cases verticales, à peine estompé par les effets de cadre auxquels se livre le bédéiste ${ }^{20}$, accélère le tempo narratif. Le textuel enfle nettement ici : récitatifs et phylactères saturent les cases, écrasant le dessin de leur poids ${ }^{21}$. C'est sur cette masse de texte que repose la cohérence de l'ensemble, car au plan iconique, la conduite de la séquence est raboteuse, heurtée, aussi chaotique que la guerre elle-même. Les gouttières isolent des images qui semblent autant de diapositives. Cette esthétique du fragment affecte la scénographie, tantôt réaliste tantôt symbolique; elle clive les espaces, ici, intimes, là, ouverts ; elle préside, enfin, au hiatus stylistique, avec la case qui reproduit une affiche républicaine en reprenant à son compte sa perspective outrée (218-4).

À l'opposé, la strate supérieure se distingue par sa cohérence graphique. De page en page, T. Modotti y déambule dans Guernica. Le tableau se déploie en une vaste tapisserie, qui flotte en suspension au-dessus de la chaîne du récit. Mêlant le visible (le tableau) et le lisible (le poème) sur le mode de la citation, la séquence affiche la filiation du neuvième art, qui puise à la double source de la littérature et des arts picturaux. Dans la conjonction des figures effarées de Guernica et des fragments du poème élégiaque de César Vallejo, l'Espagne en guerre apparaît comme ce lieu où, entre 1936 et 1939, se joue le sort de la démocratie. Dans cet hommage nourri par la peinture et la poésie de combat, la guerre civile se trouve exhaussée à un plan symbolique. La fresque réaffirme la noblesse de la cause républicaine, par-delà l'évocation, dans l'étage inférieur, des querelles idéologiques intestines et des trahisons qui contribuèrent à la chute de la République. Dans la conclusion de la partie inférieure, quelques mots d'Antonio Machado font écho à ceux de Vallejo. Également écartelés entre la foi et le désespoir, ces deux poèmes nous font appréhender l'œil-soleil de Picasso comme un signe d'espérance, à même, peut-être, de conjurer le désastre qu'il surplombe (fig. 7). 
Fig. 7. Planche 222.

\section{comme Vallejo, comme Machado - est aussi une façon d'être en prise avec les mouvements du monde.}

trompée en abandonnant la photographie, de ce que faire œuvre - comme Picasso, 
Fig. 8. Planche 82, vignette 1.

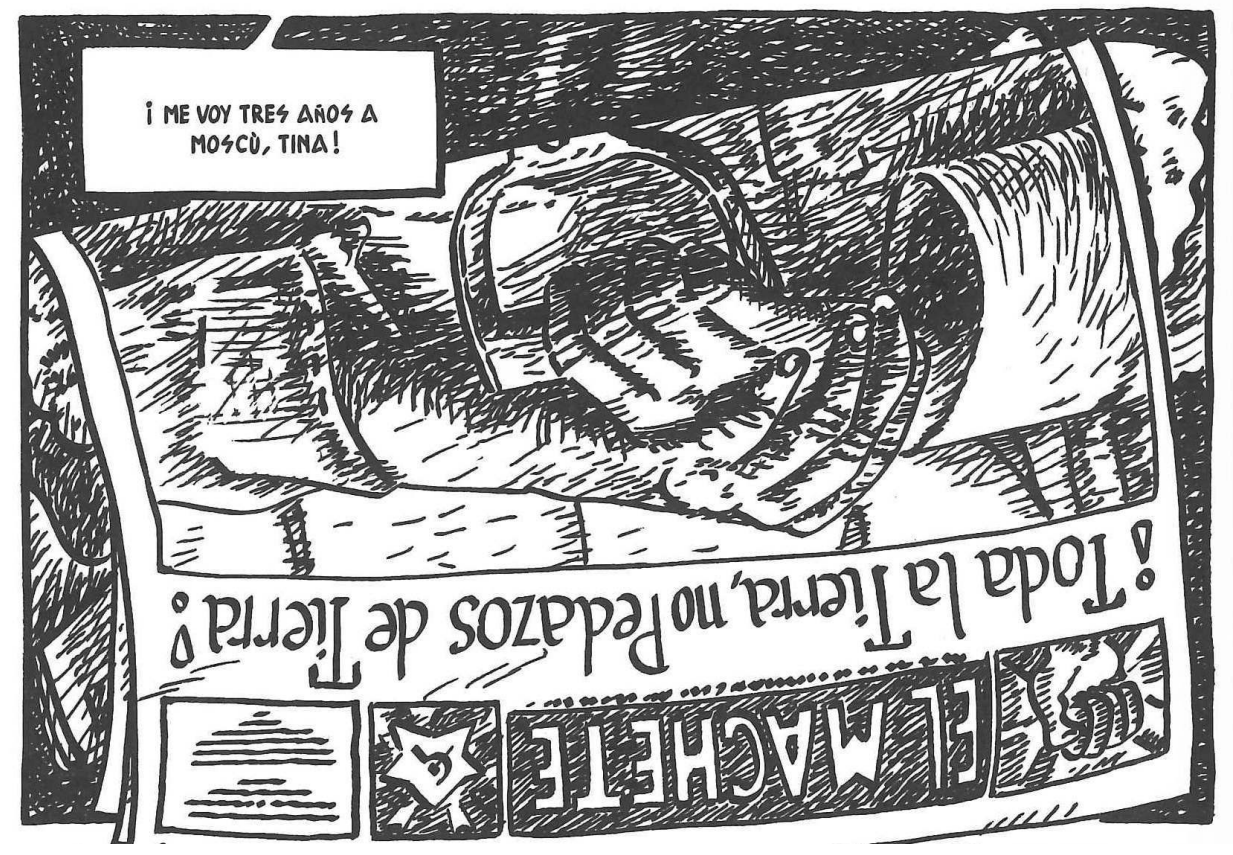

Le personnage mis en scène, dont on ne voit que les mains, est le sculpteur mexicain Xavier Guerrero. Sa posture reproduit en réalité celle du sujet de la photo Homme lisant «El Machete»; le journal apparaît pour sa part, avec ses gros titres, dans un cliché de la même série intitulé Hommes lisant "El Machete »; l'illustration, enfin, est un « re-dessin » de Mains reposant sur un outil.

Le bédéiste insère souvent dans l'espace vignettal des sujets appartenant à des photographies traitées in absentia ${ }^{24}$. Utilisées comme un simple matériau prographique, refaçonnées, les photos d'origine se font presque oublier. Ainsi en va-t-il de celle de l'enfant posant devant des figuiers de barbarie, prise lors d'un voyage que réalisèrent Modotti et Weston pour les besoins d'un livre, en 1926. La continuité narrative de la séquence dans laquelle s'intègre cette œuvre est assurée par la voiture dans laquelle voyagent Modotti et Weston. La logique de tressage et le recadrage de la photo conduisent logiquement le lecteur, lorsqu'il en arrive à cette case, à se centrer sur le véhicule du deuxième plan. En sorte que la photographie redessinée de l'enfant aux cactus se fond dans le décor (fig. 9). 
Fig. 9. Planche 44 , vignette 8.

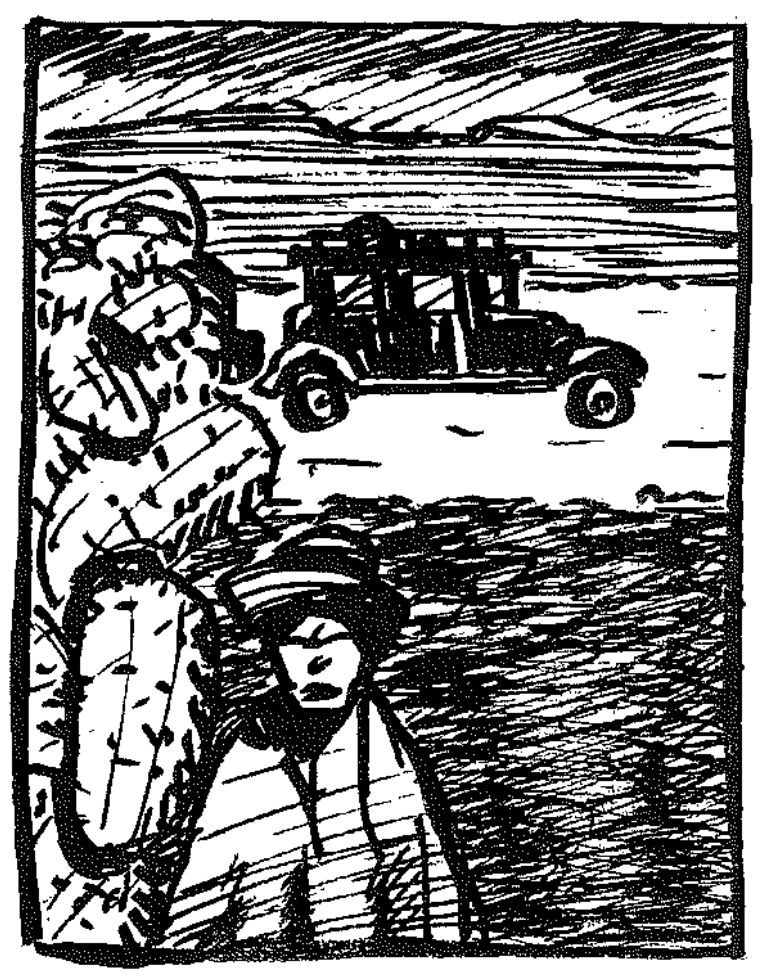

Morceau de réalité arraché au continuum temporel, la photographie, bien qu'elle témoigne du réel, a quelque chose d'une unité mutilée. Et l'usage qu'en fait cette bande dessinée est à double tranchant. D'un côté, en dépit de la stylisation que le dessinateur leur impose, les clichés redessinés renforcent l'effet de réel. Comme c'est le cas dans Le Photographe (Guibert et Lefèvre 2012), qui incorpore des épreuves au récit bédéistique, ils asseyent la prétention de véridicité du récit biographique. Les photos de Modotti ou de Weston insérées dans le récit ont une valeur indicielle ${ }^{25}$. Elles attestent leur existence à tous deux, comme sujets photographiques et comme opérateurs. Mais en les enserrant dans une séquence bédéistique, en leur restituant un hors-champ, en leur réinjectant de la temporalité, le dessinateur les réinvente. Garantes du sérieux du discours biographique, elles deviennent paradoxalement des leviers de la mise en fiction et rendent à l'auteur toute sa liberté de créateur.

41 La photographie, enfin, comme pratique artistique, occupe une place non négligeable dans la diégèse. Prises, développées, commentées, les photos adoptent la forme d'insistants parallélépipèdes, abîmés dans des vignettes qui rédupliquent l'effet-cadre (fig. 10). 
Fig. 10. Planche 33, vignettes 2-3.
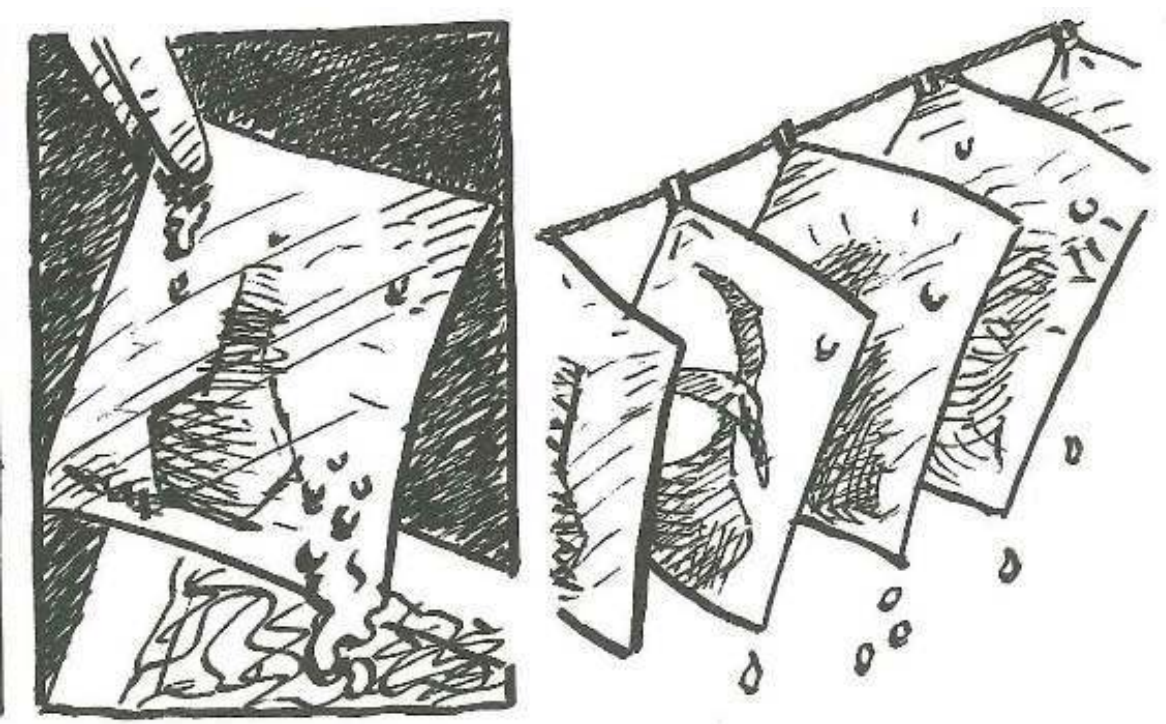

42 Ainsi, l'absorption des photographies redessinées et l'omniprésence du rectangle - métonymie et matrice de l'art de Modotti et de l'art de la bande dessinée - réaffirment silencieusement mais continûment, sur un mode purement iconique, la force de la relation biographique.

Même si l'œuvre s'appuie sur l'ensemble des propriétés du texte-image pour se faire le laboratoire d'une réflexion tantôt discrète, tantôt plus turbulente, sur les enjeux de la démarche biographique, c'est bien dans le niveau visuel que cette relation complexe et l'ambiguïté qui la fonde atteignent à leur pleine expression. Et au vu de la subtilité de l'imaginaire (auto)(bio)graphique que l'auteur mobilise pour en décrire les rouages, on est tenté de souscrire à l'idée que «[c'est] vraisemblablement du côté des expérimentations graphiques de la bande dessinée qu'il convient aujourd'hui de chercher les interrogations et les réponses les plus stimulantes qui empêchent le biographique de se scléroser » (Aron et Preyat 2008).

\section{BIBLIOGRAPHIE}

Altarriba, Antonio et Kim, 2016a, L'art de voler, trad. Alexandra Carrasco, Paris, Denoël Graphic. Altarriba, Antonio et Kim, 2016b, L'aile brisée, trad. Alexandra Carrasco, Paris, Denoël Graphic. Aron, Paul et Preyat, Fabrice, 2008, « Introduction », COnTEXTES. La question biographique en littérature, $\mathrm{n}^{\circ} 3$, [En ligne], https://journals.openedition.org/contextes/2543, consulté le 9 novembre 2016.

DOI : $10.4000 /$ contextes. 2543

Barthes, Roland, 1980, La Chambre claire. Note sur la photographie, Paris, Seuil. 
Bourdieu, Pierre, 1986, «L'illusion biographique », Actes de la recherche en sciences sociales, vol. 62, $\mathrm{n}^{\circ}$ 1, p. 69-72.

Broqua, Vincent et Marche, Guillaume, 2010, « Introduction : L'épuisement du biographique ? ", dans V. Broqua et G. Marche (dir.), L'Épuisement du biographique?, Newcastle, Cambridge Scholars Publishing, p. 1-21.

Davodeau, Étienne, 2005, Les Mauvaises Gens : une histoire de militants, Paris, Delcourt.

Dosse, François, 2010, « Préface : L'explosion biographique », dans V. Broqua et G. Marche (dir.), L'Épuisement du biographique?, Newcastle, Cambridge Scholars Publishing, p. XI-XVI.

Guibert, Emmanuel et Lefèvre, Didier (2012-2014), Le Photographe, t. 1, 2 et 3, Marcinelle/Paris, Dupuis, coll. « Aire Libre ».

Groesteen, Thierry, 1999, Système de la bande dessinée, Paris, PUF, coll. « Formes Sémiotiques ».

Groesteen, Thierry, 2011, Bande dessinée et narration. Système de la bande dessinée 2, Paris, PUF, coll. Formes Sémiotiques.

Jablonka, Ivan, 2012, Histoire des grands-parents que je n'ai pas eus, Paris, Seuil, coll. « La librairie du $\mathrm{XXI}^{\mathrm{e}}$ siècle ".

Jablonka, Ivan, 2014a, « Histoire et bande dessinée », La Vie des idées.fr, 18 novembre 2014, [En ligne], http://www.laviedesidees.fr/Histoire-et-bande-dessinee.html, consulté le 14 décembre 2016.

Jablonka, Ivan, 2014b, L'histoire est une littérature contemporaine, Paris, Seuil, coll. « La librairie du $\mathrm{XXI}^{\mathrm{e}}$ siècle ».

Madelénat, Daniel, 1984, La Biographie, Paris, PUF, coll. « Littératures Modernes ».

Regard, Frédéric, 2002, " L'Éthique du biographique. Réflexions sur une tradition britannique », Littérature, vol. 128, $\mathrm{n}^{\circ}$ 4, p. 80-92.

Spiegelman, Art, [1987-1991] 2005, Maus, un survivant raconte, t. 1 et 2, trad. Judith Ertel, Paris, Flammarion.

\section{Corpus}

De la Calle, Ángel, [2003-2005] 2011a, Modotti. Una mujer del siglo veinte, édition revue et élargie, Madrid, Sinsentido.

De la Calle, Ángel, 2011b, Tina Modotti, trad. Rachel Viné-Krupa, Paris, Vertige Graphic.

\section{NOTES}

1. Citations textuelles et renvois seront adossés à l'édition française (2011b), sauf mention contraire. Pour des questions de propriété intellectuelle, les illustrations sont quant à elles tirées de l'édition espagnole.

2. Le photographe américain, qui avait eu Tina Modotti pour modèle avant de la former à la photographie, vécut et travailla à ses côtés au Mexique, jusqu'en 1927.

3. «Toute histoire serait littérature? Aucune histoire ne serait littérature ? La seule manière d'échapper à ce balancement stérile, c'est de faire en sorte que l'aspiration littéraire du chercheur ne soit pas un renoncement, une récréation après le "vrai" travail, un repos du 
guerrier, mais un bénéfice épistémologique; qu'elle signifie progrès réflexif, redoublement d'honnêteté, surcroît de rigueur, mise au jour du protocole, discussion des preuves, invitation au débat critique. [...] Il ne s'agit pas de tuer l'histoire à coups de fiction et de rhétorique, mais de la retremper par une forme, une construction narrative, un travail sur la langue, dans un texteenquête qui épouse son effort de vérité. La création littéraire est l'autre nom de la scientificité historienne » (2014b : 13-14).

4. On désignera comme métabiographiques ces épisodes qui opèrent une mise à distance du récit biographique et commentent la genèse de l'œuvre.

5. Dans ce registre, on ne peut manquer de songer à Maus (Spiegelman 2005). Plus récemment, dans le champ hispanique, on mentionnera le diptyque d'Altarriba et Kim $(2009,2016)$. En France, Étienne Davodeau revient sur l'engagement politique de ses parents dans une bande dessinéereportage extrêmement fouillée : Les Mauvaises Gens (2005).

6. «C'était comme ne trouver aucun palmier, aucune oasis, aucune terre promise après avoir erré quarante ans durant dans un désert atroce » (25-5).

7. Á. De la Calle ne la désigne d'ailleurs jamais ainsi, et si Paco Ignacio Taibo II emploie le terme dans le prologue original du deuxième volume (267-268), il se montre plus prudent dans le prologue écrit pour les besoins de l'édition française : «Ce livre est, et n'est pas, une biographie » (5).

8. En témoigne le séminaire "Les écritures visuelles de l'histoire de la bande dessinée ", accueilli en 2015 à la BNF, ou la tenue d'un colloque à Pau, en 2011, sur le sujet de la bande dessinée historique.

9. Voir la planche intégralement consacrée à une biographie antérieure de T. Modotti (12).

10. «[D]e par le travail de la lacune, le biographique est présent dans l'absence qu'elle signifie. En effet, plutôt que de ne rien signifier, l'absence qui la définit fait signe pour l'auteur et le critique » (Broqua et Marche $2010: 14$ ).

11. Toutes les illustrations sont tirées de Modotti. Una mujer del siglo veinte (Sinsentido, 2011).

12. On entend par là l'espace qui s'ouvre au-delà de l'hypercadre, cette ligne, continue ou non, qui constitue le tracé extérieur de la planche.

13. Voir 157-5, par exemple.

14. «[L]e personnage biographique n'est pas la vérité qui aurait dégénéré en une fiction, mais une fiction qui se hisserait au niveau d'une "vérité". [...] [L]a fabulation biographique nous projette inévitablement dans le champ d'un savoir: elle ouvre ce que Jacques Rancière nomme une "poétique du savoir" [...]. C'est toute l'éthique de la fabulation, qui n'est pas affabulation» (Regard $2002: 88$, l'auteur souligne).

15. «Tout biographe, soumis et rebelle à la fois, résurrectionniste et embaumeur, sauve et tue ; il viole, transgresse l'intime et interdite clôture de l'autre, mais, en même temps, il est exproprié et cancérisé par l'emprise d'un “corps étranger” sur son psychisme » (Madelénat 1984 : 91).

16. Ma traduction.

17. Ma traduction.

18. La spatialité joue un rôle déterminant dans la concaténation des deux lignes narratives, comme dans ces pages où la plaque de rue du niveau diégétique ressurgit dans le méta-niveau (105-4 et 106-4).

19. «Dans laquelle de ces rues, qui s'apprêtaient à connaître des tourments inimaginables, Tina décida-t-elle que la photographie n'était pas ce qu'il y avait de plus important dans sa vie?» (154-7) ; «Je m'imagine ces printemps à Paris... / et je rêve de Tina rattrapée par la nostalgie... marchant d'un pas mélancolique le long de la rive gauche de la Seine » (194-1).

20. Dans cette séquence, le dessinateur joue plus volontiers encore que dans le reste de l'œuvre sur la délimitation de la vignette. Partiellement virtuelle, elle aère le multicadre. De la Calle pratique ainsi tantôt la clôture à bord perdu, tantôt la bordure feinte - les bulles rectangulaires 
marquant les contours de la case-, tantôt l'insertion de cadres internes, pour délimiter un espace iconique au sein d'une case prise d'assaut par le texte.

21. L'auteur se livre par exemple à une longue énumération pour évoquer les combattants étrangers qui rejoignent les Brigades internationales (219-3). Ailleurs, c'est l'espace iconique luimême qui est envahi par la liste des participants au $2^{\mathrm{e}}$ Congrès international des écrivains pour la défense de la culture de 1937, qui sert de décor à une vignette (222-5).

22. «Entourée de photographies qui justifient la majuscule au mot Art, se trouvait la magnifique image prise par Tina » (61).

23. «Elle donna une autre lecture photographique de la misère du Mexique, qu'elle n'avait perçue jusqu'alors que comme élément du décor » (78-4).

24. C'est le cas de Flor de manita, photographie à laquelle renvoie la case 67-6. Une vendeuse y présente une fleur à Modotti. L'artificialité de la composition (mise en scène de la monstration, jeu de clair-obscur) alerte au reste le lecteur quant à la probabilité d'un jeu intericonique.

25. "Dans la photographie, je ne puis jamais nier que la chose a été là. Il y a double position conjointe : de réalité et de passé. Et puisque cette contrainte n'existe que pour elle, on doit la tenir, par réduction, pour l'essence même, le noème de la Photographie »(Barthes 1980 : 120).

\section{RÉSUMÉS}

L'article a pour objet la biographie dessinée Tina Modotti, d'Ángel De la Calle (2011), qui retrace les vingt dernières années $\mathrm{du}$ parcours de la photographe italienne et militante communiste éponyme (1896-1942).

$\mathrm{Au}$ plan narratif, la ligne biographique se double d'une ligne secondaire, alternativement autobiographique et autofictionnelle, au sein de laquelle l'auteur se projette dans un double de papier, pour revenir sur la genèse de l'œuvre. On s'attachera à cerner la manière dont l'imaginaire et la personne du dessinateur travaillent et trouent la matière biographique, et on se demandera dans quelle mesure la biographie devient ici scène du biographe.

Pour ce faire, on reviendra sur le fonctionnement du niveau biographique, où malgré le recours à la première personne le récit s'efforce à l'objectivité. On s'intéressera au rôle de l'imaginaire graphique dans le développement d'une forme de médiation documentaire. La deuxième ligne narrative, qui signe par instants une véritable entrée en régime de fiction, sera au cœur de la seconde partie de l'analyse. Après avoir étudié ces deux aspects de l'œuvre, on percevra mieux la portée des choix esthétiques de l'auteur, car l'imaginaire graphique est en réalité, plus fondamentalement, au service de la relation biographique et de sa dynamique identificatoire.

The object of the present study is Ángel De la Calle's Tina Modotti (2011). This graphic biography tells the story of the eponymous Italian photographer and communist activist (1896-1942).

On a narrative level, the biographical line is coupled with a secondary line, alternately autobiographical and autofictional, where the author projects himself into a drawn fictional double to evoke the genesis of his work. I will attempt to examine how both versions of the author coexist with the biographical material and to what extent the biography serves, in this specific context, as a stage for the biographer.

To do so, I will begin by analyzing the mechanisms of the biographical level since, despite the use of the first person singular, efforts are made to keep the narrative as objective as possible. I will also discuss the role of graphic imagination in transmitting documentary information. I will then 
focus on the second narrative line which, at times, drifts onto the universe of fiction. Finally, studying those two aspects will allow to perceive the significance of the author's esthetic choices because his graphic imagination is fundamentally serving the biographical relationship and its identifying dynamic.

INDEX

Mots-clés : bande dessinée, De la Calle (Ángel), Modotti (Tina), biographie, autobiographie, photographie, imaginaire graphique, relation biographique

Keywords : comics, De la Calle (Ángel), Modotti (Tina), biography, autobiography, photography, graphical imagination, author/character relationship

\section{AUTEUR}

\section{MARIE-CAROLINE LEROUX}

Université de Limoges

, EHIC 